\title{
Examination of Potential Measures of Vine Maturity in Potato
}

\author{
Emily Haga $^{1}$, Brooke Weber ${ }^{1}$, Shelley Jansky ${ }^{1,2}$ \\ ${ }^{1}$ Department of Horticulture, University of Wisconsin, Madison, USA; ${ }^{2}$ United States Department of Agriculture-Agricultural Re- \\ search Service (USDA-ARS), Albany, USA. \\ Email: shjansky@wisc.edu
}

Received December $8^{\text {th }}, 2011$; revised January $11^{\text {th }}, 2012$; accepted February $20^{\text {th }}, 2012$

\begin{abstract}
Plant maturity is a complex physiological trait routinely evaluated by plant breeders because of its agronomic, economic, and breeding implications. In potato, plant maturity is typically estimated by monitoring vine characteristics. This study investigates several reported measures of vine maturity in potato cultivars, including examination of flower development, leaf chlorophyll content, and leaf peroxidase activity to see what method is most appropriate for maturity classification in temperate production regions. These three measures were evaluated multiple times throughout a single growing season across two locations. The data were analyzed using canonical discriminant and dichotomous tree analyses. Both methods revealed that flower development is not an accurate indicator of maturity, even though it is a common component of maturity screening protocols. Peroxidase measures were slightly better, but the optimal period of time to assess this trait was variable across locations and a considerable time commitment is required to collect and process samples. Monitoring leaf chlorophyll content throughout plant development was most appropriate for predicting vine maturity under these conditions, as this trait showed the most consistency and greatest prediction accuracy $(69 \%$ $71 \%$ ) relative to peroxidase activity and flowering development. Additionally, chlorophyll measurements are a more practical method of measuring maturity due to the ease of data collection. Leaf chlorophyll content best distinguished late cultivars from early-medium and medium-late cultivars. However, it did not separate early-medium from mediumlate cultivars. Combining chlorophyll monitoring with peroxidase and flowering measures improved the ability to distinguish among the early-medium and medium-late maturity classes. However, doing so only increased classification accuracy by $3 \%$.
\end{abstract}

Keywords: Solanum tuberosum; Plant Maturity; Peroxidase; Leaf Chlorophyll; Flower

\section{Introduction}

In potato, cultivars are classified into maturity types according to the length of the growing season required to produce a harvestable product. This information provides growers with an estimate of an appropriate harvest date. Physiologically, a potato crop is considered mature once tubers have finished bulking (growing in size) and the skin has set (the periderm is developed) [1]. Variability among cultivars for the number of days from planting to maturity has led to the designation of early, medium, late, and very late maturity classes. Knowledge about maturity class is a critical aspect of commercial potato production. Therefore, breeders evaluate this trait when developing new cultivars. Nevertheless, there is no standard classification scheme used by potato breeders to characterize the maturity class of a cultivar [2-17].

In large part, a standard maturity measure in potato is lacking because tubers are produced underground, and monitoring the below ground status of a plant part presents many challenges. However, the production of tubers is associated with many changes at the whole plant level, including altered morphology (i.e. reductions in node number, axillary branching, leaf development, flowering, and fruit set), increased rates of photosynthesis, and adjustment of source/sink relationships as tubers expand and demand assimilates [7,18]. Rather than relying on destructive field harvest, potato breeders instead assess maturity class by monitoring physiological changes in the potato vine.

This study investigates the utility of three reported vine maturity scoring methods on potato cultivars grown under long-day conditions in Wisconsin: flower development, leaf chlorophyll content, and leaf peroxidase activity. Flowering is a physiological attribute commonly utilized by potato breeders for evaluating maturity [2, 4-6]. Abundant flowering has been correlated with delayed tuber bulking [7]. Additionally, the chlorophyll 
content of leaves has been found to decrease as plants senescence. Consequently, leaf chlorophyll may provide an alternative measure of maturity [18-20]. Biochemical markers associated with maturity may also provide a widely applicable alternative for objectively classifying plant material. Recently, peroxidase (POX) has been evaluated in relation to maturity type for a number of crops since it is found in a wide variety of plant tissues and is connected with many stress and maturity related traits [13-17]. The goal of this research was to provide information that will aid breeders in selecting the most appropriate method for maturity classification in temperate production regions.

\section{Materials and Methods}

\subsection{Plant Material}

Six potato clones were used as reference standards for this study. An effort was made to select clones that are familiar to the potato industry and that fall into welldefined maturity classes. However, conflicting maturity designations were reported for some cultivars [22-25]. In general, the cultivars fell into three maturity classes, defined as early-medium (Russet Norkotah and Atlantic), medium-late (Pike and C75-5 + 297), and late (Snowden and Russet Burbank). Tubers of each standard were provided by the Wisconsin Seed Potato Certification State Farm, except clone C75-5 + 297 (297), which is a breeding line developed from a somatic hybrid of $S$. tuberosum and S. brevidens [26] that had been maintained clonally in the field.

\subsection{Field Design}

Clones were evaluated at two locations: Hancock Agricultural Research Experiment Station in Hancock, WI and Eagle Heights Research Plots in Madison, WI. Tuber pieces were started in the greenhouse six to eight weeks prior to transplanting in the field at Hancock on June 4 and at Eagle Heights on June 15, 2009. Each plot consisted of two seed pieces of a clone; plots were replicated three times in a randomized complete block design (RCBD), with $30 \mathrm{~cm}$ spacing between plants in a plot and $60 \mathrm{~cm}$ spacing between plots. Rows were $90 \mathrm{~cm}$ apart. Standard cultural practices for potato production were used at Hancock. These practices were mimicked at Eagle Heights as closely as possible. However irrigation, fertilization, and pest management procedures varied due to differences in infrastructure, pest pressure, and availability of fungicide and fertilizer products.

\subsection{Maturity Assessment}

Over the course of the field season (mid July to late September), several methods were used to assess maturity.
The timeline for evaluations at each location is summarized for all variables in Table 1. Each variable was assessed as follows:

Flowering: Flowering was scored every $7-14$ days on a 0 - 3 scale: $0=$ not flowering, $1=$ flower buds present, 2 = flowers present, and 3 = post-flower. This information was used to calculate initial and final dates of flowering, as days after planting (dap), and then used to determine the duration of the flowering period (in days).

Chlorophyll: Leaf chlorophyll content was determined on four dates throughout the growing season at approximately14 day intervals using a chlorophyll meter (Minolta SPAD-502), a non-destructive instrument commonly used to determine nitrogen levels by providing a SPAD (an indexed chlorophyll content reading) measurement $[21,27,28]$. Readings were taken at a central point between the midrib and leaf margin on the terminal leaflet of the third to fourth fully expanded leaf of the plant. Duplicate measurements were made on both plants in a plot, and averages of these four measurements were taken to account for variability in chlorophyll. For each plot, a mean of all chlorophyll content readings collected across the season was also calculated.

Peroxidase: Peroxidase was examined at three time points during the season at approximately 14 day intervals by collecting the same leaves that were used to measure chlorophyll content. For each sample, 0.15 to $0.20 \mathrm{~g}$ of fresh leaf material was added to a $2 \mathrm{ml}$ tube containing a $4 \mathrm{~mm}$ stainless steel grinding ball (OPS Diagnostics, Lebanon, $\mathrm{NJ}$ ), frozen in liquid nitrogen, and stored at $-80^{\circ} \mathrm{C}$ until the assays could be performed.

Peroxidase was assayed using a modified protocol from Malik and Singh [15] and Sandhu et al. [14], scaled down in volume to increase its high-throughput capacity by making it amenable to a 96-well plate. Leaf tissue was macerated in $1.7 \mathrm{ml}$ of $0.1 \mathrm{M}$ cold phosphate buffer $(\mathrm{pH}$ 6.5), (68.5 ml 0.2 $\mathrm{M} \mathrm{NaH}_{2} \mathrm{PO}_{4}, 31.5 \mathrm{ml} 0.2 \mathrm{M} \mathrm{Na}_{2} \mathrm{HPO}_{4}$,

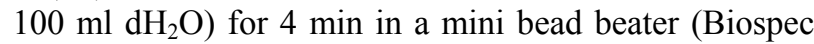
Products, Inc., Bartleville, OK). The supernatant was collected into a clean tube after centrifuging at $4{ }^{\circ} \mathrm{C}$ for $25 \mathrm{~min}$ at $15,000 \mathrm{x} \mathrm{g}$ and put on ice for use as the enzyme extract. For each sample, $7.5 \mu \mathrm{l}$ of the enzyme extract was added to a single well on a UV plate, along with 262 $\mu 10.1 \mathrm{M}$ cold phosphate buffer and $15 \mu \mathrm{l}$ o-dianisidine $(2.93 \mathrm{mg} / \mathrm{ml}$ methanol). Immediately before taking an absorbance reading, $15 \mu 1$ of $0.2 \mathrm{M}$ hydrogen peroxide

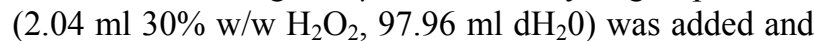
mixed by pipetting. The reaction progress was monitored by measuring absorption at $430 \mathrm{~nm}$ at $30 \mathrm{sec}$ intervals for 3 min in a Biotek Synergy HT microplate reader (Winooski, VT). The program KC4 version 3.4 (Biotek) was used to calculate the maximum rate of reaction $\left(\mathrm{V}_{\max }\right)$, which is a measure of enzymatic activity that is directly proportional to the amount of enzyme present [15]. The 
Table 1. Misclassification error rates calculated from dichotomous tree analyses to assess the accuracy of each variable in predicting maturity class. Leaf chlorophyll content, flower development, and leaf peroxidase activity were examined for individual score dates (dap = days after planting) and as combined estimates (Mean, Initial, Final, and Duration).

\begin{tabular}{|c|c|c|c|c|c|}
\hline \multicolumn{3}{|c|}{ Hancock } & \multicolumn{3}{|c|}{ Eagle Heights } \\
\hline Variable & dap $^{1}$ & Misclassification Rate & Variable & $\operatorname{dap}^{2}$ & Misclassification Rate \\
\hline \multirow{5}{*}{ Chlorophyll } & 40 & $36 \%$ & \multirow{5}{*}{ Chlorophyll } & 37 & $35 \%$ \\
\hline & 55 & $36 \%$ & & 52 & $44 \%$ \\
\hline & 69 & $31 \%$ & & 66 & $53 \%$ \\
\hline & 83 & $43 \%$ & & 80 & $41 \%$ \\
\hline & Mean & $31 \%$ & & Mean & $29 \%$ \\
\hline \multirow{13}{*}{ Flowering } & 35 & $53 \%$ & \multirow{13}{*}{ Flowering } & 30 & $59 \%$ \\
\hline & 40 & $58 \%$ & & 37 & $59 \%$ \\
\hline & 55 & $67 \%$ & & 46 & $65 \%$ \\
\hline & 62 & $67 \%$ & & 52 & $65 \%$ \\
\hline & 69 & $50 \%$ & & 66 & $47 \%$ \\
\hline & 76 & $50 \%$ & & 73 & $47 \%$ \\
\hline & 83 & $53 \%$ & & 80 & $44 \%$ \\
\hline & 88 & $58 \%$ & & 87 & $53 \%$ \\
\hline & 100 & $67 \%$ & & - & - \\
\hline & 112 & $67 \%$ & & - & - \\
\hline & Initial & $56 \%$ & & Initial & $59 \%$ \\
\hline & Final & $42 \%$ & & Final & $42 \%$ \\
\hline & Duration & $47 \%$ & & Duration & $42 \%$ \\
\hline \multirow{3}{*}{ Peroxidase } & 40 & $42 \%$ & \multirow{3}{*}{ Peroxidase } & 37 & $41 \%$ \\
\hline & 55 & $31 \%$ & & 52 & $44 \%$ \\
\hline & 69 & $42 \%$ & & 66 & $38 \%$ \\
\hline
\end{tabular}

${ }^{1}$ Hancock Planting Date $=$ June 4, 2009; ${ }^{2}$ Eagle Heights Planting = June 15, 2009.

weight of each leaf sample was used to calculate the peroxidase activity so it could be expressed as $\Delta \mathrm{Amin}^{-1}$. $\mathrm{g}^{-1}$ fresh weight. Peroxidase activity was determined three times for each sample and averaged to give one value.

\subsection{Statistical Analysis}

Tables and graphs were made in Microsoft Excel (2007) to visualize variation within and among locations and cultivars for all variables throughout the season. The predictive power of each variable was examined by developing diagnostics keys in R version 2.10.0 [29] using a dichotomous tree approach. The trees allowed the overall accuracy of each variable at both locations to be determined, which was done by calculating a misclassification error rate (number of incorrectly classified cultivars/the total number of cultivars evaluated) (Table 1).

Canonical discriminant analyses (CDA) were also con- ducted in SAS ${ }^{9} 9.2$ [30] to determine if certain variable combinations would further discriminate the three maturity classes at each location. In this approach, a first and second canonical variable were identified and then visualized using scatter plots (Figure 1). The CDA variables were combined by location and subjected to the dichotomous tree approach to determine whether combining variables decreased misclassification rates as compared to misclassification rates of individual variables (Figure 2). Other combinations of variables were also analyzed to determine whether a low misclassification rate could be obtained (data not shown).

\section{Results}

Flowering, chlorophyll, and peroxidase measurements taken throughout the season were examined graphically at each location to visualize patterns that might associate with maturity class designations (Figures 3 and 4). At 


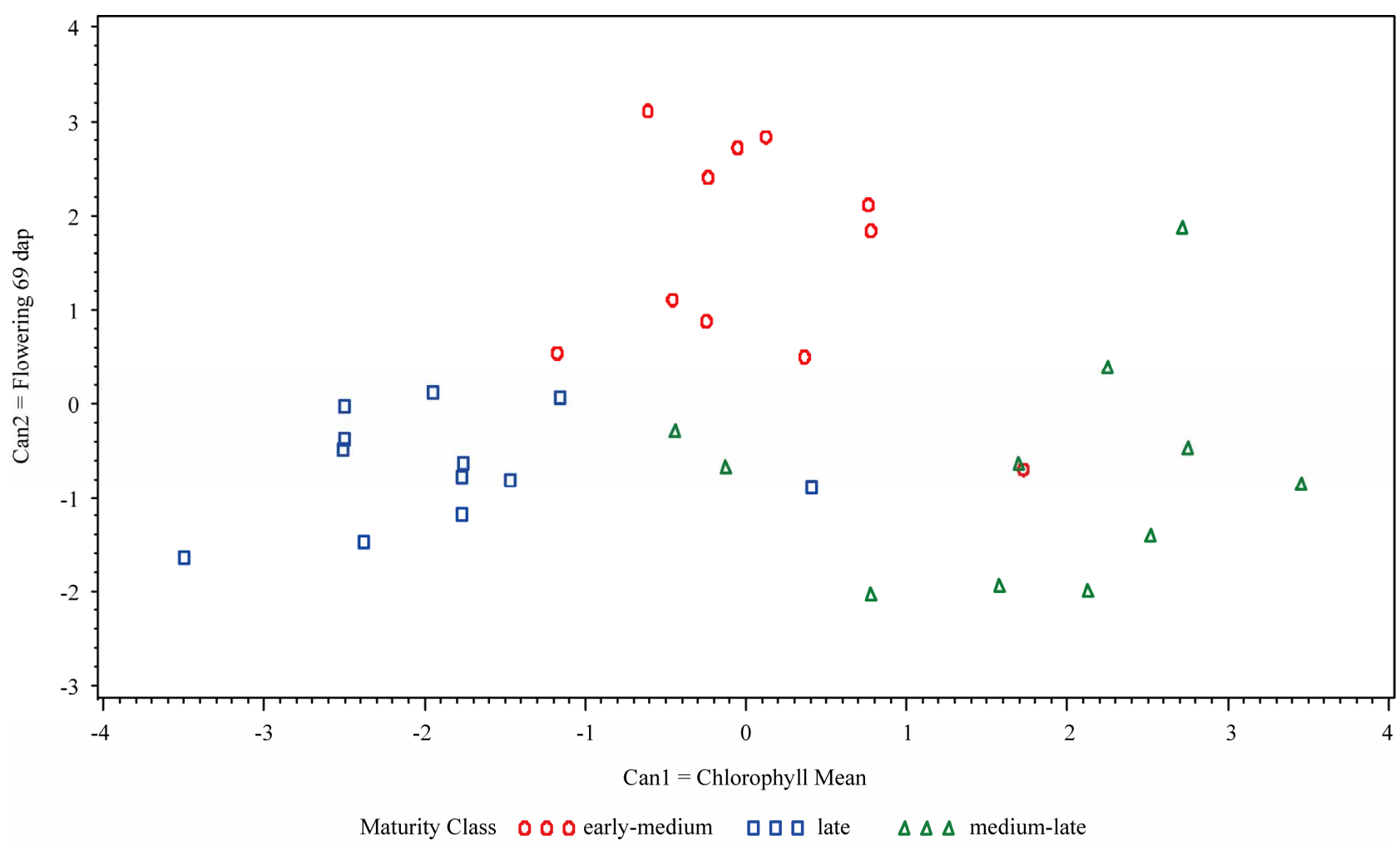

(a)

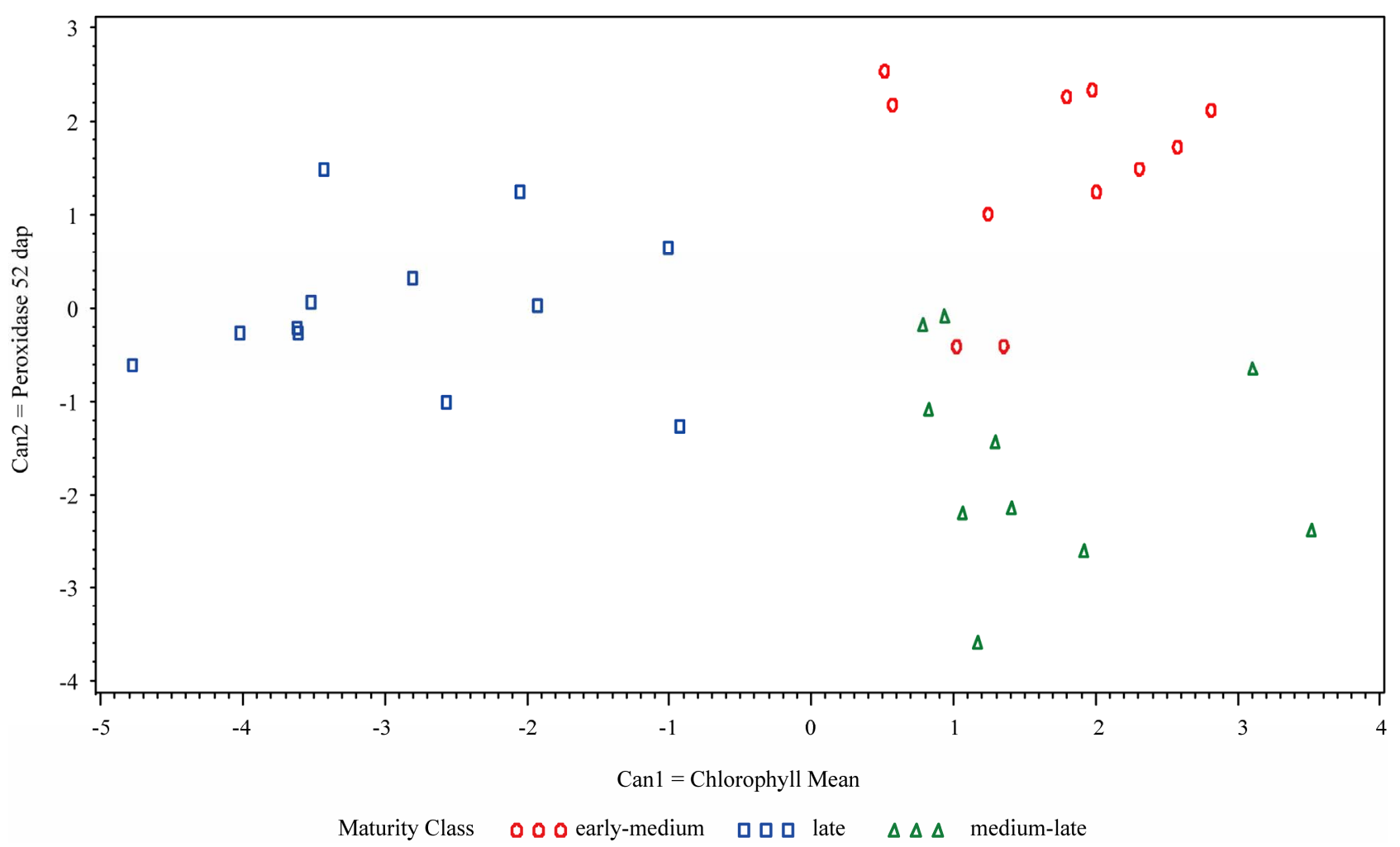

(b)

Figure 1. Canonical Discriminant Analysis of the two variables that best separated maturity classes (early-medium, late and medium-late) at (a) Hancock: Can1 = mean of all chlorophyll measurements and Can2 = flowering development 69 dap and (b) at Eagle Heights: Can1 = mean of all chlorophyll measurements and Can2 = peroxidase activity 52 dap. 


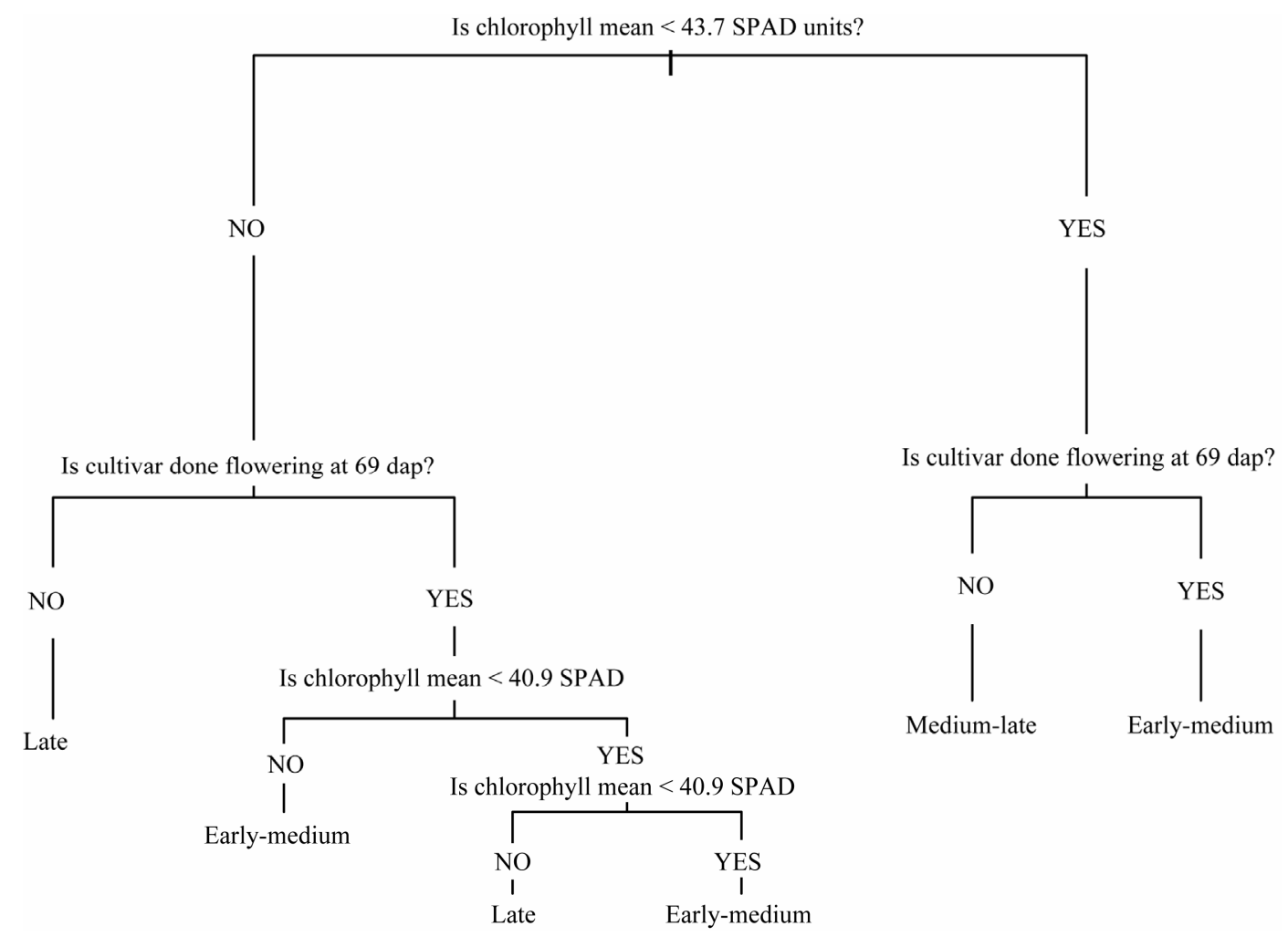

(a)

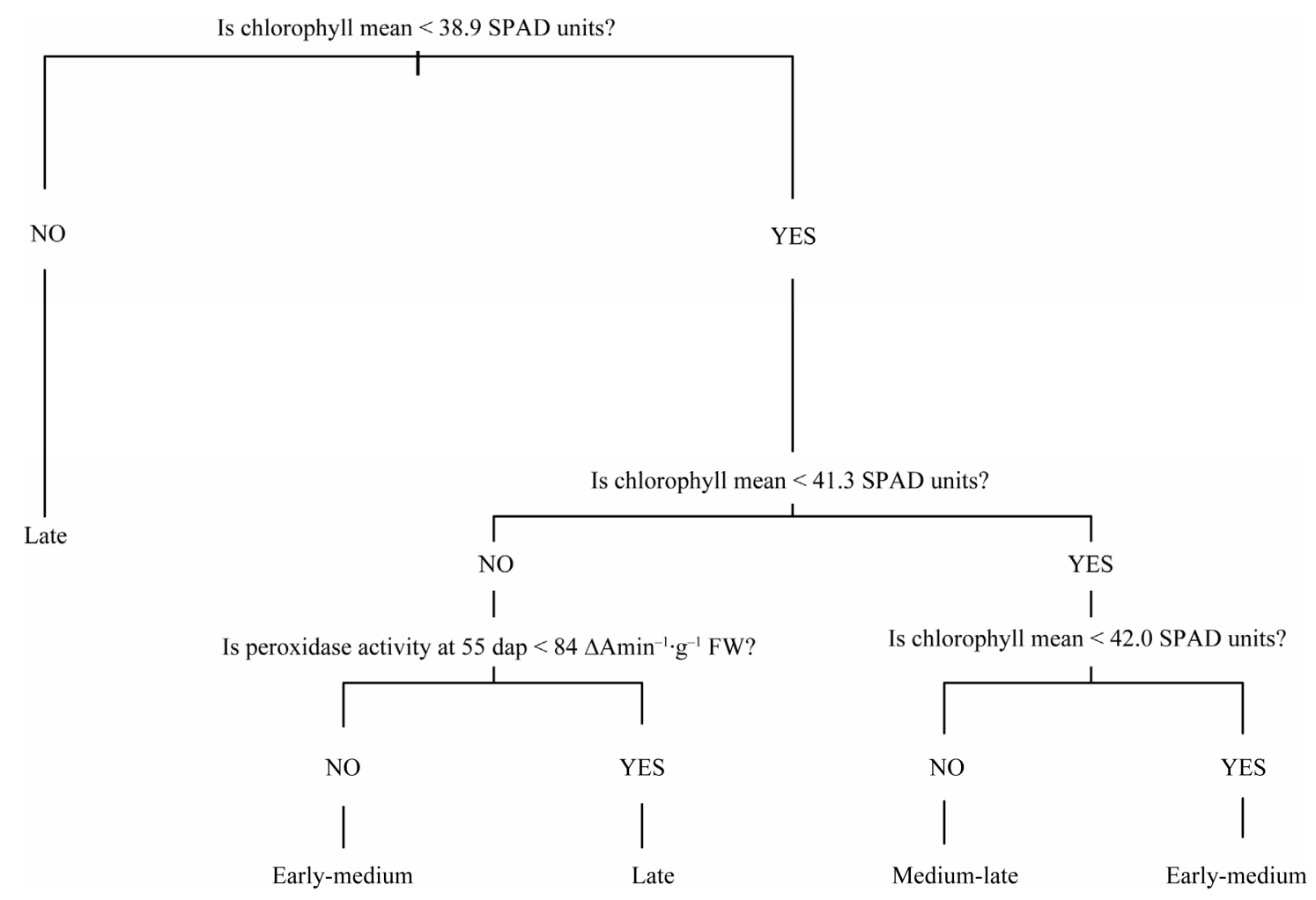

(b)

Figure 2. Dichotomous tree diagnostics keys for maturity classification using the first and second canonical variables identified at each location by canonical discriminant analyses. (a) Hancock (mean leaf chlorophyll content and flowering development 69 days after planting); (b) Eagle Heights (mean leaf chlorophyll and peroxidase activity 55 days after planting). 


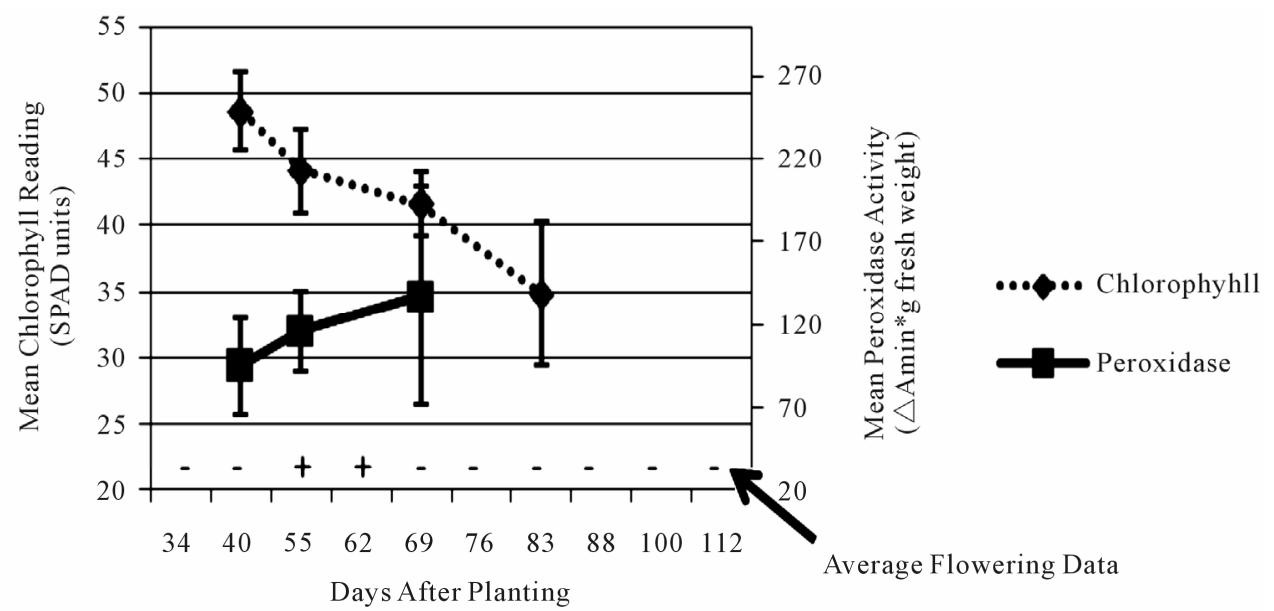

(a)

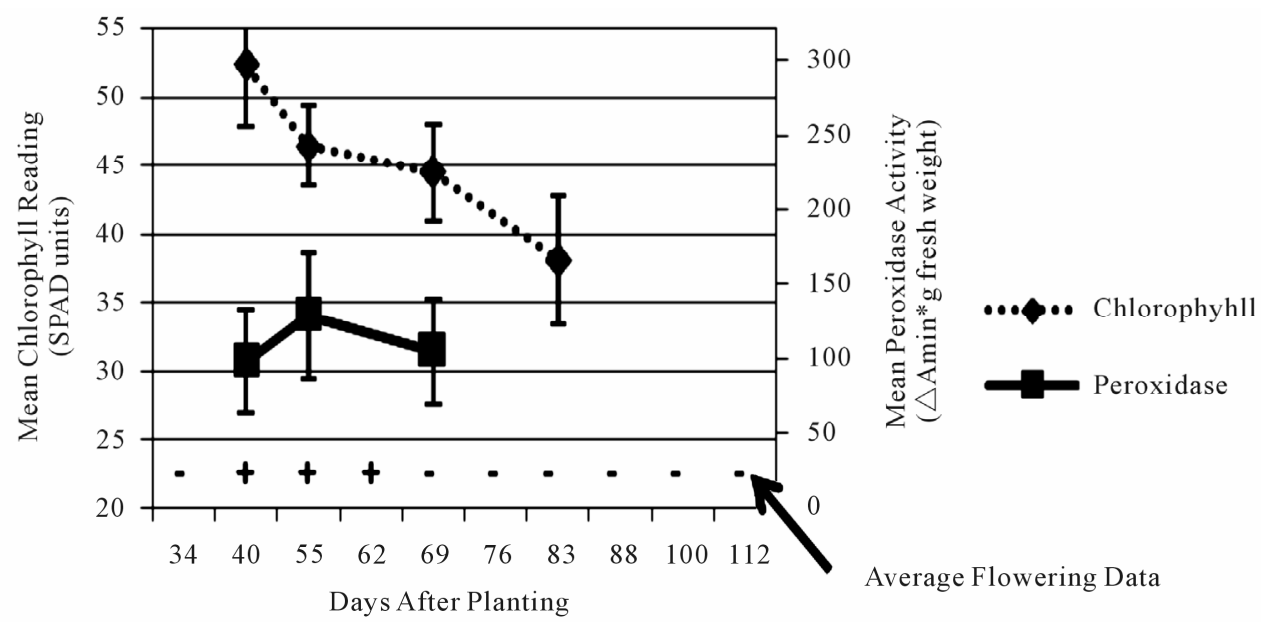

(b)

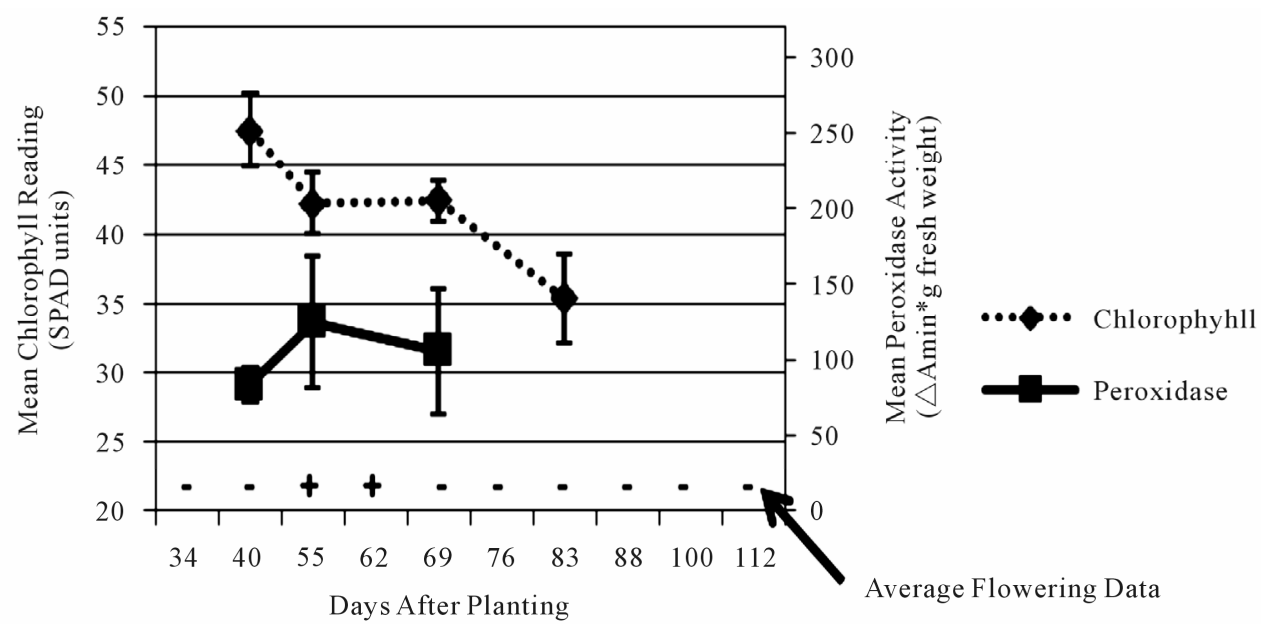

(c)

Figure 3. Average leaf chlorophyll content (dashed line), leaf peroxidase level (solid line), and stage of flower development (flowers present $=+$ /flowers absent $=-$ ) values for early-medium maturing varieties "Russet Norkotah" and "Atlantic" (a), medium-late maturing varieties "Pike” and “297” (b), and late maturing varieties "Snowden” and "Russet Burbank" (c) at Hancock. 


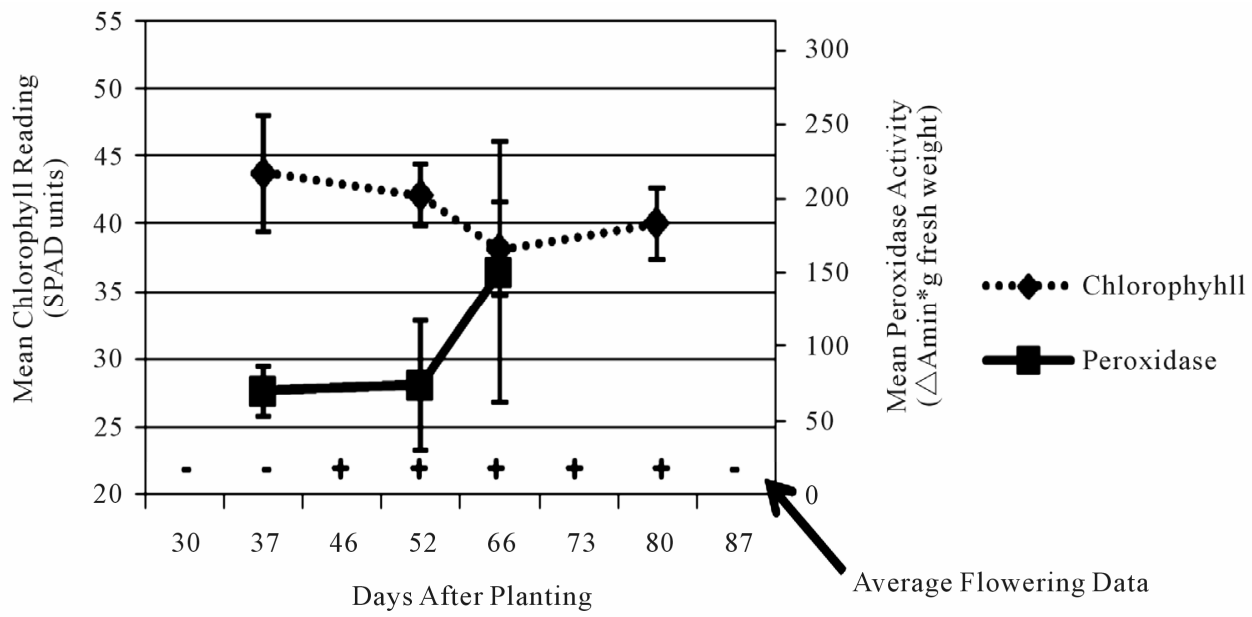

(a)

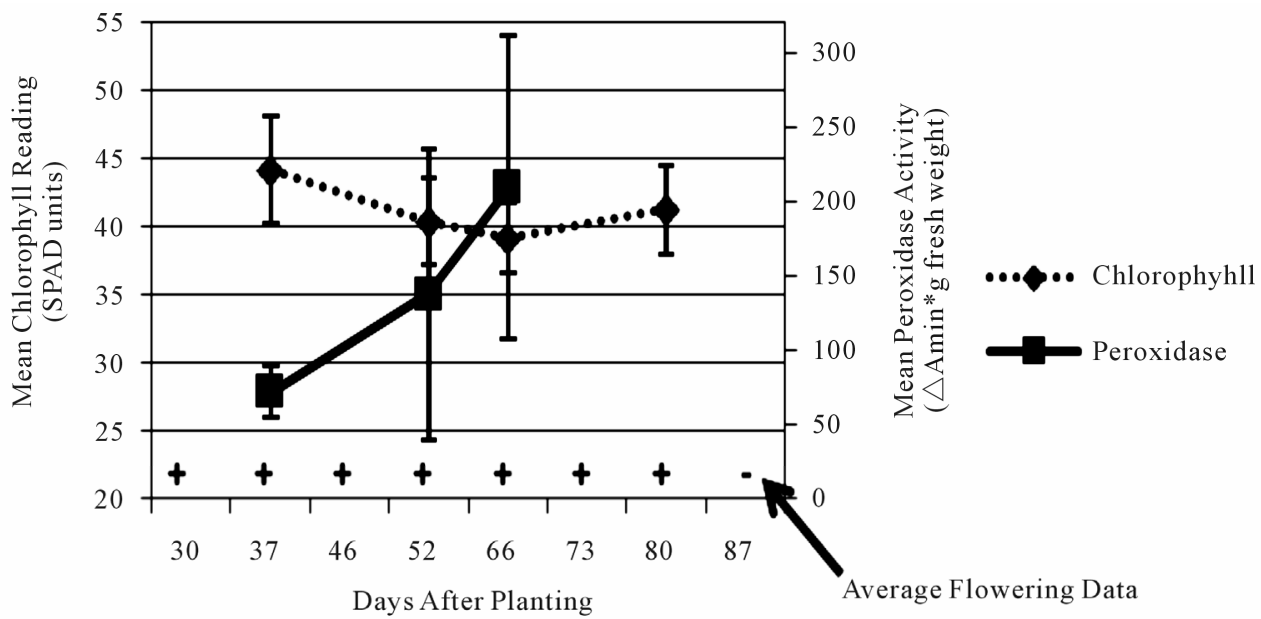

(b)

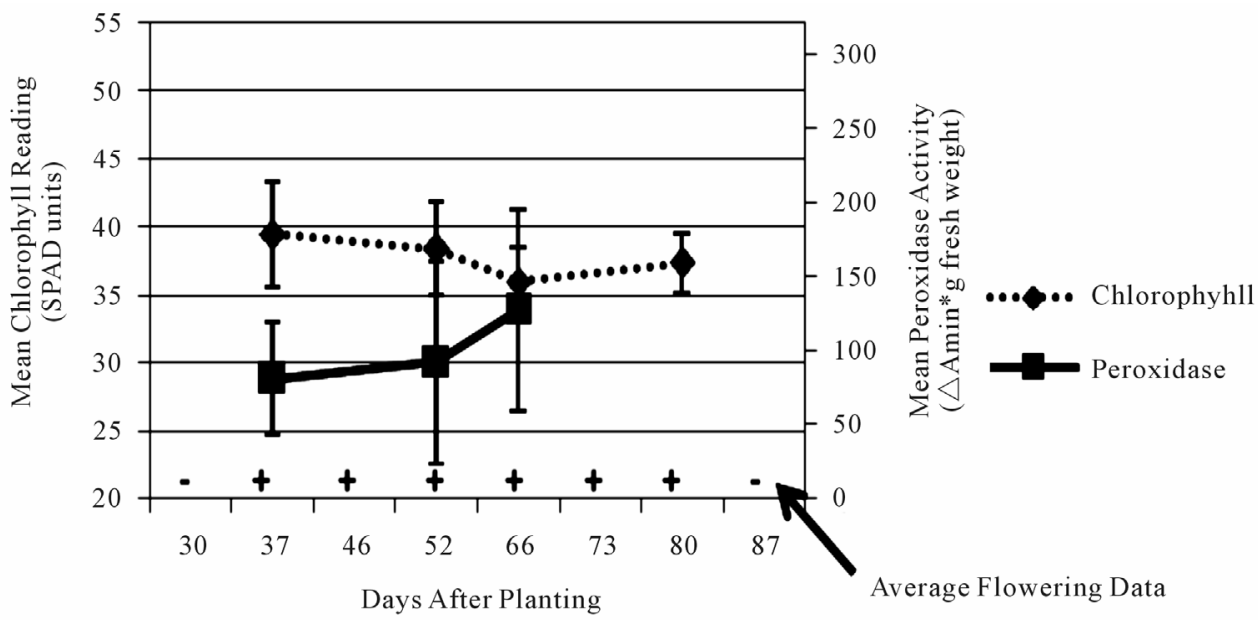

(c)

Figure 4. Average leaf chlorophyll content (dashed line), leaf peroxidase level (solid line) and stage of flower development (flowers present $=+$ /flowers absent $=-$ ) values for early-medium maturing varieties "Russet Norkotah" and "Atlantic" (a), medium-late maturing varieties "Pike" and "297" (b), and late maturing varieties "Snowden" and "Russet Burbank" (c) at Eagle Heights. 
each time point, chlorophyll readings showed the most consistency. Initial chlorophyll contents ranged among maturity classes from 47 - 52 SPAD units at Hancock and 40 - 44 SPAD units at Eagle Heights. At both locations, the late maturity class had the lowest initial reading, followed by the medium-late, then early-medium classes. As the season progressed, chlorophyll levels decreased for all three maturity classes at both locations, but a steeper decline was observed at Hancock (13 SPAD units) than at Eagle Heights (3 SPAD units).

In contrast to chlorophyll content, peroxidase activity was highly variable for each cultivar, but in general it increased over time (Figures 3 and 4). Average peroxidase activity was initially around 83,98 , and $95 \Delta \mathrm{Amin}^{-1} \cdot \mathrm{g}^{-1}$ fresh weight for the late, medium-late, and early-medium maturity classes, respectively, at Hancock. By the end of the season, peroxidase activity had increased to 105,105 , and $138 \Delta \mathrm{Amin}^{-1} \cdot \mathrm{g}^{-1}$ fresh weight for the late, medium-late, and early-medium classes, respectively (Figure 3). At Eagle Heights, average peroxidase activity started out lower than Hancock (about $81 \Delta \mathrm{Amin}^{-1} \cdot \mathrm{g}^{-1}$ fresh weight for the late maturity class and $70 \Delta \mathrm{Amin}^{-1} \cdot \mathrm{g}^{-1}$ fresh weight for the early-medium and medium-late categories), but ended higher for the medium-late and late maturity classes (209 and $127 \Delta \mathrm{Amin}^{-1} \cdot \mathrm{g}^{-1}$ fresh weight, respectively), and roughly the same for the earlymedium class (150 $\Delta \mathrm{Amin}^{-1} \cdot \mathrm{g}^{-1}$ fresh weight) (Figure 4).

Like peroxidase activity, flower development was also highly variable throughout the season. For example, inconsistencies of as much as 44 days (6 - 7 weeks) were observed in flowering duration among plants of a single cultivar at a single location due to variation in initial and final flowering dates (data not shown). However, when average flowering development scores were considered at each time point, different patterns among maturity classes became evident at both locations (Figures 3 and 4). At Hancock, flowering began 40 dap on average for the medium-late maturity class and lasted approximately 22 days, while for the early-medium and late classes it began 55 dap on average and lasted approximately 7 days (Figure 3). In contrast, flowering at Eagle Heights occurred earlier than at Hancock and lasted much longer (Figure 4). On average, the medium-late class flowered first (30 dap), followed by the late maturity class (37 dap), then the early-medium class (46 dap). Flowering ended around the same time for all three maturity classes, leading to a final duration of 50,43 , and 34 days, respectively.

The first and second variables (Can1 and Can2) in the canonical discriminant analysis (CDA) at Hancock were the mean of all chlorophyll measurements (Cmean) and flowering development 69 dap, respectively. Cmean best discriminated among the three maturity classes, while flowering development 69 dap further distinguished early-medium from the two other classes (Figure 1). At Eagle Heights, Cmean was also the most influential variable (Can1); however the second canonical variable (Can2) was peroxidase activity 52 dap. Cmean separated the late maturity class from the other two classes, while peroxidase activity 52 dap further distinguished the early-medium and medium-late categories.

Misclassification rates from the individual variable dichotomous tree analyses ranged from $31 \%-67 \%$ at Hancock and 29\% - 65\% at Eagle Heights (Table 1). Chlorophyll and peroxidase measurements provided the greatest accuracy. In particular, Cmean was more precise in distinguishing categories at both locations. Combining the first and second canonical variables into a more complex tree increased the accuracy by $3 \%$ at both locations, to $28 \%$ at Hancock and $26 \%$ at Eagle Heights. The diagnostics keys generated from these complex trees are shown in Figure 2. For comparison, other variables were also combined to form a more complex tree to see if this would further improve accuracy. However, doing this did not lower the misclassification rates.

\section{Discussion}

When a cultivar is released, it is assigned a maturity class based on observations of vine growth and tuber bulking characteristics over many years. Breeders do not have this much information in early generations, so it would be useful to find a technique that can predict maturity class. The measures of maturity examined in this study did not correlatestrongly with the maturity class designations of the reference cultivars used. However, mean leaf chlorophyll content across the season (Cmean) and peroxidase activity did show some promise in their ability to classify maturity. In particular, monitoring leaf chlorophyll content throughout the season and averaging those values provided the highest prediction accuracy. Some of the individual chlorophyll and peroxidase measurements also provided comparable accuracy to Cmean, but the optimal single score dates were not consistent across locations.

Cmean was best able to separate the late maturity class from the other two classes. The late maturity class generally had a lower chlorophyll content throughout the season as compared to the early-medium and medium-late classes. At Hancock, this difference became smaller over time and was not as large as at Eagle Heights, which is why the Cmean misclassification rate was slightly higher at Hancock (31\% vs. $29 \%)$. The observation that late cultivars could be distinguished early in the season indicates that chlorophyll screening at the beginning of the season may allow for early detection of late clones. This would be a valuable selection tool for breeders interested in avoiding late material. Further studies monitoring 
chlorophyll content in more detail may reveal whether even earlier detection is possible.

Canonical discriminant analysis revealed that combining the mean chlorophyll content (Cmean) with other measures increased the ability to distinguish among the early-medium and medium-late maturity classes. However, dichotomous tree analysis revealed that doing this increased accuracy by only 3\%. Other combinations of variables were also analyzed (data not shown), but did not result in lower misclassification rates than those selected by CDA. An additional complication with this data set is that the early-medium and medium-late classes overlapped. Consequently, the maturity classes are not physiologically distinct entities and are likely to be influenced by environmental variables.

Trends in leaf chlorophyll content were consistent across locations. At both locations, a steady decrease in chlorophyll content was observed throughout the course of plant development. This observation has also been made in several other studies $[17,19,21,27,28]$. The magnitude of the decline, however, was smaller at Eagle Heights than at Hancock, likely due to a lack of vine senescence observed at Eagle Heights. This may have resulted from variation in environmental conditions and agricultural practices between Eagle Heights and Hancock, such as soil composition (silt loam vs. sandy loam), irrigation infrastructure (hand and ground application vs. overhead), photoperiod $\left(43^{\circ} 4^{\prime} 23^{\prime \prime} \mathrm{N}\right.$ and $\left.44^{\circ} 8^{\prime} 1^{\prime \prime} \mathrm{N}\right)$, and type of fertilizer (20-10-2 vs. 28-0-0 urea and ammonium nitrate), respectively. We speculate that the environmental conditions at Eagle Heights were more conducive to prolonged plant growth than at Hancock. McGrady and Ewing [19] also noted a correlation between increasing foliar senescence and decreasing leaf chlorophyll content in potato.

In contrast to chlorophyll content, peroxidase activity increased as the season progressed at both locations. Other studies conducted in China and India have shown peroxidase activity to peak early to mid-season, and then decline roughly around the time of true seed production in soybean and tuber bulking in potato $[14,17]$. Tuberization occurred for all cultivars in this study, so it is not clear why we did not observe the same trend. The potato study conducted in India occurred under short days (9.75 $11.5 \mathrm{hr}$ photoperiod), while ours occurred under long days (12.5 - $15.5 \mathrm{hr}$ photoperiod). Other stresses in the previous studies may have also influenced peroxidase activity.

The large variability in flowering development resulted in extremely high misclassification rates and made it difficult to discern consistent trends. The final incidence of flowering and the duration of flowering period had misclassification rates comparable to some of the chlorophyll and peroxidase measurements. However, the prediction accuracy of the other flowering measures examined (i.e. initiation of flowering and individual flowering scores) was no better than random assignments of maturity designations to each variety would have been. Flowering is a photoperiod dependent process. It is believed that photoperiod is sensed by phytochrome $\mathrm{b}$, which represses flowering time genes (StSP6A and StSP3D) under long photoperiods and activates them under short photoperiods [31,32]. The flowering time genes are expressed in the leaves and their products are transported to shoot apical meristems to induce flowering. It is interesting that one of the flowering time genes (StSP6A) induces tuberization at the same time it induces flower initiation. Consequently, observations of flower production should predict tuberization. However, tuber bulking rate varies among clones and this trait is not likely controlled by flowering time genes.

The results of our study highlight the difficulty that exists in trying to assess the maturity class of potato clones using correlated traits. We examined three reported measures of vine maturity, and found that none of them accurately predicted maturity class. Although high levels of misclassification were observed for all three measures, some were more accurate than others. We found measures of flowering development to be highly inaccurate, even though they are a common component of various maturity screening protocols [2,4-6]. Peroxidase measures were slightly better, but the optimal period of time to assess this trait was variable across locations. Furthermore, a considerable time commitment is required to collect and process samples when determining peroxidase levels, making it a less desirable method of measuring maturity. Monitoring of chlorophyll throughout the season showed the most promise for researchers wishing to assess maturity of cultivated potatoes in temperate production regions. Chlorophyll measurements are also a more practical method of measuring maturity due to the ease of obtaining measurements in a short period of time. Thus, monitoring of chlorophyll is a more desirable method for assessing maturity type because it is easier to implement in breeding programs and more accurate than monitoring of flowering development and peroxidase activity. However, a better understanding of the physiological responses associated with plant aging in potato is still needed, and further research may lead to better methods of screening.

\section{REFERENCES}

[1] R. E. Voss, "Potato," In: The Agricultural Handbook Number 66: The Commercial Storage of Fruits, Vegetables, and Florist and Nursery Stocks, United States Department of Agriculture-Agricultural Research Services (USDA-ARS), 2005.

http://www.ba.ars.usda.gov/hb66/114potato.pdf 
[2] D. R. Douglas and J. J. Pavek, "Screening Potatoes for Field Resistance to Early Blight," American Potato Journal, Vol. 49, No. 1, 1972, pp. 1-9.

doi:10.1007/BF02862935

[3] C. A. Bormann, et al., "Tagging Quantitative Trait Loci for Maturity-Corrected Late Blight Resistance in Tetraploid Potato with PCR-Based Candidate Gene Markers," Molecular Plant Microbe Interactions, Vol. 17, No. 10, 2004, pp. 1126-1138.

doi:10.1094/MPMI.2004.17.10.1126

[4] A. Johanson and H. D. Thurston, "The Effect of Cultivar Maturity on the Resistance of Potatoes to Early Blight Caused by Alternaria solani," American Potato Journal, Vol. 67, No. 9, 1990, pp. 615-623. doi:10.1007/BF03043447

[5] J. Sliwka, et al., "Tagging QTLs for Late Blight Resistance and Plant Maturity from Diploid Wild Relatives in a Cultivated Potato (Solanum tuberosum) Background," Theoretical and Applied Genetics, Vol. 115, No. 1, 2007, pp. 101-112. doi:10.1007/s00122-007-0546-9

[6] S. H. Jansky, A. Hamernik and P. C. Bethke, "Germplasm Release: Tetraploid Clones with Resistance to Cold-Induced Sweetening," American Journal of Potato Research, Vol. 88, No. 3, 2010, pp. 218-225. doi:10.1007/s12230-011-9186-3

[7] P. C. Struik, M. H. P. W. Visker, J. B. Pauwels and L. T. Colon, "Are Cuttings Suitable for Assessing Maturity Type in Potato (Solanum tuberosum)?" Annals of Applied Biology, Vol. 147, No. 1, 2005, pp. 27-34. doi:10.1111/j.1744-7348.2005.00008.x

[8] H. J. van Eck, "Localisation of Morphological Traits on the Genetic Map of Potato Using RFLP and Isozyme Markers," Thesis, Landbouw universiteit, Wageningen, 1995.

[9] I. Simko, "Comparative Analysis of Quantitative Trait Loci for Foliage Resistance to Phytophthora infestans in Tuber-Bearing Solanum Species," American Journal of Potato Research, Vol. 79, 2002, pp. 125-132. doi:10.1007/BF02881521

[10] R. Zhang, "Genetic Characterization and Mapping of Partial Resistance to Early Blight in Diploid Potato," Ph.D. Thsis, Pennsylvania State University, University Park, 2004, p. 158.

[11] M. H. P. W. Visker, L. C. P Keizer, H. J. Van Eck, E. Jacobsen, L. T. Colon and P. C. Struik, "Can the QTL for Late Blight Resistance on Potato Chromosome 5 Be Attributed to Foliage Maturity Type," Theoretical and Applied Genetics, Vol. 106, No. 2, 2003, pp. 317-325.

[12] M. H. P. W. Visker, et al., "Correlation between Late Blight Resistance and Foliage Maturity Type in Potato," Euphytica, Vol. 137, No. 3, 2004, pp. 311-323. doi:10.1023/B:EUPH.0000040451.21852.d8

[13] A. L. Adám, C. S. Bestwick, B. Barna and J. W. Mansfield, "Enzymes Regulating the Accumulation of Active Oxygen Species during the Hypersensitive Reaction of Bean to Pseudomonas syringae pv. Phaseolicola," Planta, Vol. 197, No. 2, 1995, pp. 240-249.

[14] S. K. Sandhu, et al., "Peroxidase as a Biochemical Marker of Maturity Levels in Potato (Solanum tuberosum)
Cultivars under Short Days," New Zealand Journal of Crop and Horticultural Science, Vol. 35, No. 1, 2007, pp. 171-175. doi:10.1080/01140670709510181

[15] C. P. Malik and M. B. Singh, "Plant Enzymology and Histo-Enzymology: A Text Manual," Kalyani Publishers, New Delhi, 1980, p. 434.

[16] T. Janda, G. Szalai, O. V. Rios-Gonzalez and E. Paldi, "Comparative Study of Frost Tolerance and Antioxidant Activity in Cereals," Plant Science, Vol. 164, No. 2, 2003, pp. 301-306. doi:10.1016/S0168-9452(02)00414-4

[17] J. Fu, B. Huang and G. Zhang, "Physiological and Biochemical Changes during Seed Filling in Relation to Leaf Senescence in Soybean," Biologia Plantarium, Vol. 43, No. 4, 2000, pp. 545-548.

[18] E. E. Ewing, "Cuttings as Simplified Models of the Potato Plant," In: P. H. Li, Ed., Potato Physiology, Academic Press, Orlando, 1985, pp. 153-207.

[19] J. J. McGrady and E. E. Ewing, "Potato Cuttings as Models to Study Maturation and Senescence," Potato Research, Vol. 33, No. 1, 1990, pp. 97-108. doi:10.1007/BF02358134

[20] P. L. Minotti, D. E. Halseth and J. B. Sieczka, "Field Chlorophyll Measurements to Assess the Nitrogen Status of Potato Varieties," HortScience, Vol. 29, No. 12, 1994, pp. 1497-1500.

[21] E. J. Botha, B. J. Zebarth and B. Leblon, "Non-Destructive Estimation of Potato Leaf Chlorophyll and Protein Contents from Hyperspectral Measurements Using the Prospect Radiative Transfer Model," Canadian Journal of Plant Science, Vol. 86, 2006, pp. 279-291. doi:10.4141/P05-017

[22] R. E. Webb, et al., "Atlantic: A New Potato Variety with High Solids, Good Processing Quality, and Resistance to Pests," American Potato Journal, Vol. 55, No. 3, 1978, pp. 141-145. doi:10.1007/BF02852087

[23] R. L. Plaisted, et al., "Pike: A Full Season Scab and Golden Nematode Resistant Chipstock Variety," American Journal of Potato Research, Vol. 75, No. 3, 1998, pp. 117-120. doi:10.1007/BF02895845

[24] J. Stark, D. Westermann and L. T. Hopkins, "Nutrient Management Guidelines for Russet Burbank Potatoes," Extension Bulletin 840, Moscow, University of Idaho Extension, Idaho, 2004.

[25] W. Hamester and U. Hils, "World Catalogue of Potato Varieties," Agrimedia GmbH, Bergen, Germany, 2003.

[26] A. L. Tek, W. R. Stevenson, J. P. Helgeson and J. Jiang, "Transfer of Tuber Soft Rot and Early Blight Resistances from Solanum brevidens into Cultivated Potato," Theoretical and Applied Genetics, Vol. 109, No. 2, 2004, pp. 249-254. doi:10.1007/s00122-004-1638-4

[27] E. J. Botha, B. Leblon, B. Zebarth and J. Watmough, "Non-Destructive Estimation of Potato Leaf Chlorophyll from Canopy Hyperspectral Reflectance Using the Inverted Prosail Model," International Journal of Applied Earth Observation and Geoinformation, Vol. 9, No. 4, 2007, pp. 360-374. doi:10.1016/j.jag.2006.11.003

[28] A. Ierna, "Characterization of Potato Genotypes by Chlorophyll Fluorescence during Plant Aging in a Mediterra- 
nean Environment," Photosynthetica, Vol. 45, No. 4, 2007 , pp. 568-575. doi:10.1007/s11099-007-0097-y

[29] R Development Core Team, "R: A Language and Environment for Statistical Computing," R Foundation for Statistical Computing, ISBN 3-900051-07-0, Vienna, 2008. http://www.R-project.org

[30] $\mathrm{SAS}{ }^{\circledR} 9.2$ Enhanced Logging Facilities, SAS Institute Inc., Cary, NC, USA Copyright (C) 2008.

[31] C. Navarro, J. A. Abelenda, E. Cruz-Oro, C. A. Cuellar, S.
Tamaki, J. Silva, K. Shimamoto and S. Prat, "Control of Flowering and Storage Organ Formation in Potato by Flowering Locus T," Nature, Vol. 478, No. 7367, 2011, pp. 119-123. doi:10.1038/nature10431

[32] The Potato Genome Sequencing Consortium, "Genome Sequence and Analysis of the Tuber Crop Potato," Nature, Vol. 475, No. 7355, 2011, pp. 189-195.

doi:10.1038/nature 10158 